Fig. 1. The personal protective equipment (PPE) burn rate calculator provides PPE usage rater based on healthcare provider role.

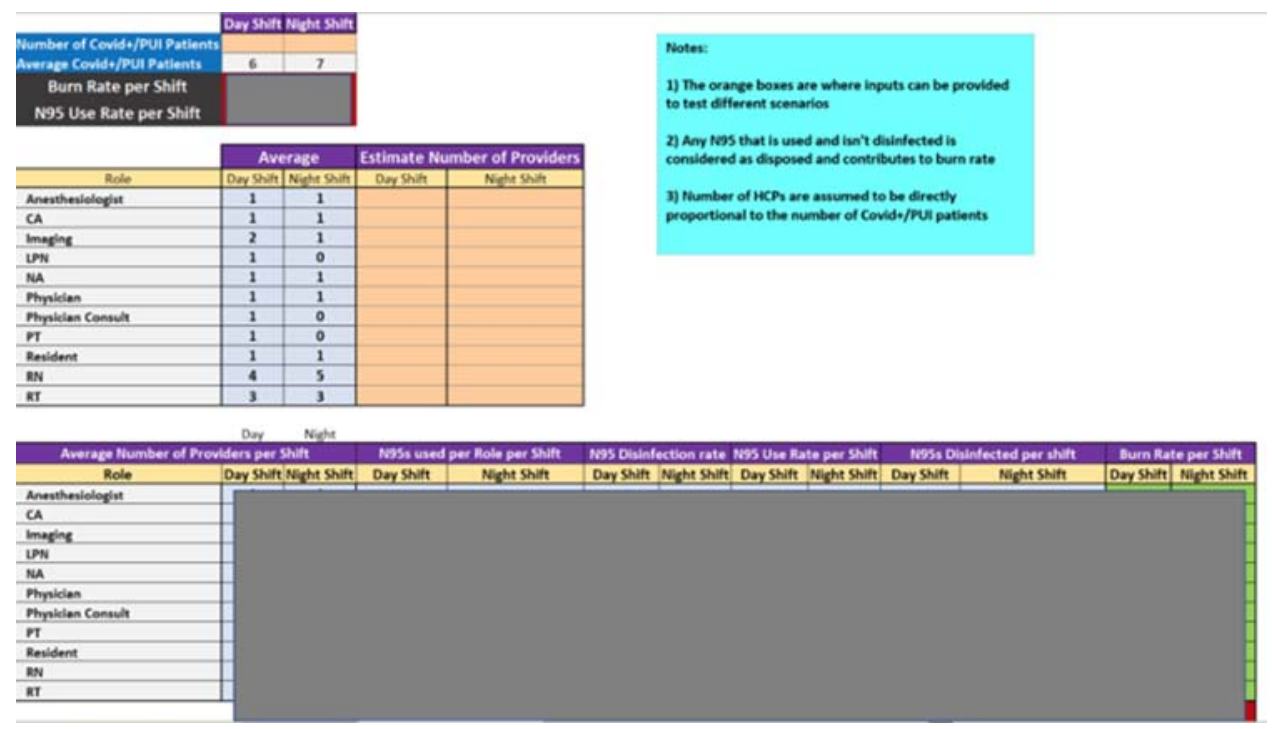

COVID-19/PUI units. We also determined our N95 use rate, which informed future planning to optimize our inventory. Based on sampled data, the calculator can estimate N95 burn rates for simulated provider and patient volumes (Fig. 1).

We have demonstrated a novel sampling methodology that can be easily implemented to aggregate PPE use data. We also developed a Microsoft Excel tool that can allow administrators to reasonably prognosticate future supply and demand for PPE. Though we only sampled one unit, this tool also can allow users to sample more units and generate tighter confidence intervals on burn and use rates. At our institution, PSOs were already stationed on units performing other tasks, which aided data collection.

By examining provider use patterns, we can implement education initiatives that target specific provider roles. Our sampling method will benefit from validation, and we intend to continue to feedback our findings in other units to refine the output. We hope that our findings can assist other hospitals in easily determining their PPE burn rates and prolong their current supplies of PPE.
Acknowledgments. We thank all providers who were willing to provide data for our sampling procedure as well as the patient safety officers that collected data on the COVID-19 wards at our institution.

Financial support. No financial support was provided relevant to this article.

Conflicts of interest. All authors report no conflicts of interest relevant to this article.

\section{References}

1. Rational use of personal protective equipment for coronavirus disease 2019 (COVID-19). World Health Organization website. https://www.who. int/publications-detail/rational-use-of-personal-protective-equipment-forcoronavirus-disease-(covid-19)-and-considerations-during-severe-shortages. Published April 6, 2020. Accessed April 29, 2020.

2. COVID-19: strategies for optimizing the supply of PPE. Centers for Disease Control and Prevention website. https://www.cdc.gov/coronavirus/2019ncov/hcp/ppe-strategy/index.html. Accessed April 16, 2020.

\title{
A coronavirus disease 2019 (COVID-19) outbreak in a hospital and hospital closure: A note
}

\author{
Rujittika Mungmunpuntipantip ${ }^{1}$ and Viroj Wiwanitkit ${ }^{2,3,4}$ \\ ${ }^{1} 26$ Medical Center, Bangkok Thailand, ${ }^{2}$ Dr DY Patil University, Pune, India, ${ }^{3}$ Hainan Medical University, Haikou, China and ${ }^{4}$ Chulalongkorn University, Bangkok \\ Thailand
}

To the Editor-The coronavirus disease 2019 (COVID-19) outbreak is a global public health problem. After its occurrence in the Republic of China in December, 2019, the disease spread

Author for correspondence: Rujittika Mungmunpuntipantip, E-mail: rujittika@ gmail.com

Cite this article: Mungmunpuntipantip R and Wiwanitkit V. (2020). A coronavirus disease 2019 (COVID-19) outbreak in a hospital and hospital closure: A note. Infection Control \& Hospital Epidemiology, 41: 1475-1476, https://doi.org/10.1017/ice.2020.194 worldwide, occurring in $>160$ countries. ${ }^{1}$ The virus is highly contagious, and close contact in crowded places is an important contributing factor to SARS-CoV-2 transmission. Medical personal are an important at-risk population that can get COVID-19 due to close contact with patients in daily practice. ${ }^{2,3}$ Sporadic case reports about COVID-19 from many countries have been published., Hospital-acquired infection is possible, as are hospital outbreaks. Here, we present data from Thailand, the second country where the disease occurred in early January $2020 .^{6}$ 
The setting is a rural district hospital in Yala province, the southernmost province of Thailand that shares an international border with Malaysia, another country where COVID-19 outbreaks occur. This small, 30-bed hospital serves local people in that rural district. The outbreak occurred on March 22, 2020, when 3 medical personnel ( 2 nurses and 1 physician) developed fever and COVID-19 was confirmed after they had provided regular care to local people with a history of COVID-19 contact. All 3 medical personnel are presently under respiratory isolation, but none has had lung complications. All of the other 21 medical personnel of the hospital, including 7 physicians, are under quarantine. The hospital had to be closed and no longer provides any service. According to our best knowledge, this is the first report of COVID-19 outbreak in a hospital that resulted in total disruption of hospital function.

The COVID-19 outbreak in hospitals is serious because it can result in abrupt cessation of local medical care and especially management of COVID-19 during the outbreak. Hospitals usually have protective systems in place, but a high-volume load might result in unsuccessful disease control. A good hospital infection control program during COVID-19 should be simple but strict, with aggressive procedures that might differ from routine clinical practice. Strategic planning to reduce unnecessary physical examinations, to implement universal drug distribution, or to postpone unnecessary procedures during the crisis period, is necessary.
Acknowledgments.

Financial support. No financial support was provided relevant to this article.

Conflicts of interest. All authors report no conflicts of interest relevant to this article.

\section{References}

1. Hsia, W. Emerging new coronavirus infection in Wuhan, China: situation in early 2020. Case Study Case Rep 2020;10:8-9.

2. Huh S. How to train the health personnel for protecting themselves from novel coronavirus (COVID-19) infection during their patient or suspected case care. J Educ Eval Health Prof 2020 [Epub 2020 Mar 7]. doi: 10.3352/ jeehp.2020.17.10.

3. Glauser W. Proposed protocol to keep COVID-19 out of hospitals. CMAJ 2020;192:E264-E265.

4. Joob B, Wiwanitkit V. COVID-19 in medical personnel: observation from Thailand. J Hosp Infect 2020;104:453.

5. Liu M, He P, Liu HG, et al. Clinical characteristics of 30 medical workers infected with new coronavirus pneumonia [in Chinese]. Zhonghua Jie He He Hu Xi Za Zhi. 2020;43:209-214.

6. Yasri S, Wiwanitkit V. Editorial: Wuhan coronavirus outbreak and imported case. Adv Trop Med Pub Health Int 2020;10(1):1-2.

\title{
Electronic screening through community engagement: A national strategic plan to find COVID-19 patients and reduce clinical intervention delays
}

\author{
Mehrdad Amir-Behghadami MSc ${ }^{1,2,3}$ and Masoumeh Gholizadeh $\mathrm{PhD}^{1,2}$ \\ ${ }^{1}$ Tabriz Health Services Management Research Center, Health Management and Safety Promotion Research Institute, Tabriz University of Medical Sciences, \\ Tabriz, Iran, ${ }^{2}$ Iranian Center of Excellence in Health Management (ICEHM), Department of Health Service Management, School of Management and Medical \\ Informatics, Tabriz University of Medical Sciences, Tabriz, Iran and ${ }^{3}$ Student Research Committee (SRC), Tabriz University of Medical Sciences, Tabriz, Iran
}

To the Editor-Coronavirus disease 2019 (COVID-19) began circulating in Wuhan, Hubei Province, China, in December 2019. Evidence of human-to-human transmission has been reported in both communities and hospitals ${ }^{1}$; COVID-19 is a highly contagious disease that can spread rapidly through respiratory droplets of infected individuals. ${ }^{2}$ According to one of the first published COVID-19 studies, the most common symptoms at onset are fever, cough, myalgia, and/or fatigue, and less common symptoms include sputum, headache, hemoptysis, and diarrhea. ${ }^{3}$ However, these symptoms may be more severe in the elderly, the immunosuppressed, and those with chronic diseases including diabetes, cardiovascular diseases, cancer, and pulmonary dysfunction. ${ }^{4,5}$

Author for correspondence: Dr Masoumeh Gholizadeh, Iranian Center of Excellence in Health Management, School of Management and Medical Informatics, Tabriz University of Medical Sciences, University Rd, Golbad, EAZN 5165665811, Tabriz, East Azerbaijan, Iran, E-mail: Gholizadehm@tbzmed.ac.ir.

Cite this article: Amir-Behghadami M and Gholizadeh M. (2020). Electronic screening through community engagement: A national strategic plan to find COVID-19 patients and reduce clinical intervention delays. Infection Control \& Hospital Epidemiology, 41: 1476-1478, https://doi.org/10.1017/ice.2020.188
The global pandemic is evolving dynamically. On January 30, 2020, the World Health Organization (WHO) declared that COVID-19 is a "Public Health Emergency of International Concern (PHEIC)" during its second meeting of the Emergency Committee. ${ }^{6}$ As of February 27, 2020, there were 1,610,909 accumulative confirmed cases and 99,690 cumulative deaths globally.? Iranian health authorities confirmed the first COVID-19 cases on February 19, 2020, in Qom. As of February 27, 2020, there had been $>66,220$ confirmed cases of COVID-19 and 4,110 deaths in Iran. ${ }^{8}$ Notably, however, Iran has had the highest improvement in COVID-19; it ranks second in the world after China. Recently, although the number of new cases reported in China has been steadily decreasing, epidemics in other countries are still a major concern. Prevention and identification of the disease have become the most important tasks in Iran, and the government has invested many material and human resources to manage the epidemic. ${ }^{9}$ Currently, no licensed preventative vaccine or specific antiviral therapy is available for COVID-19, and according to the basic theory of controlling infectious diseases, the most effective measures include eliminating the source of infection, disrupting

(c) 2020 by The Society for Healthcare Epidemiology of America. All rights reserved This is an Open Access article, distributed under the terms of the Creative Commons Attribution licence (http://creativecommons.org/licenses/by/4.0/), which permits unrestricted re-use, distribution, and reproduction in any medium, provided the original work is properly cited. 